\title{
A Survey on User Interfaces for Interaction with Human and Machines
}

\author{
Mirza Abdur Razzaq \\ Department of Computer Science \\ Shah Abdul Latif University \\ Khairpur, Pakistan \\ Muhammad Ali Qureshi \\ Department of Telecommunication Engineering \\ University College of Engineering and Technology \\ The Islamia University of Bahawalpur, Pakistan
}

\author{
Kashif Hussain Memon \\ Department of Computer Systems Engineering \\ University College of Engineering and Technology \\ The Islamia University of Bahawalpur, Pakistan \\ Saleem Ullah \\ Department of Computer Science \& IT \\ Khwaja Fareed University of Engineering and IT \\ Rahim Yar Khan, Pakistan
}

\begin{abstract}
Interaction with the machines and computers is achieved using user interfaces. Nowadays, with the tremendous growth of technology, the interaction is made more simple and flexible. The study of user interfaces for human-computers and machines interaction is the main focus of this paper. In particular, an extensive overview of different user interfaces available so far is provided. The review covers text-based, graphical-based, and new class of emerging user interfaces to interact with the machines and computers. This work will be helpful for the development of new user interfaces.
\end{abstract}

Keywords-Command line interface (CLI); graphical user interface (GUI); user interface (UI); sixth sense device; natural language interface; brain-computer interface; emerging user interfaces

\section{INTRODUCTION}

Human Machine Interaction (HMI) and Human Computer Interaction (HCI) are used for effective control of machines (e.g., automobiles, control panels to operate industrial machines), and computers. ${ }^{1}$ Other technologies used in the industry are Operator Interface Terminal (OIT) and Operator Interface Console (OIC). The interaction is achieved using user interfaces to control the input and output. The user interface (UI) is considered as prime ingredient of computer user satisfaction. The UI consists of both hardware and software. The design of an user in UI affects the amount of effort the user must spend to provide input for the system and to interpret the output of the system. Fig. 1 shows a nice example of human-machine interaction using input and output controls. The output interface is used to assist the users by displaying the feedback from the system. HMI is an interface between the human (user) and the machine (or equipment) and it is typically local to one machine or piece of equipment e.g., UI of a mechanical system, a vehicle or an industrial installation (see Fig. 2). Currently, various user interfaces exist to interact with machines and computers [1]. The equipment manufacturing companies are competing with each other by introducing innovative user interfaces to capture large market shares. Seeing the importance of user interfaces, we provide in

\footnotetext{
${ }^{1}$ https://en.wikipedia.org/wiki/User_interface
}

this paper, an overview of the existing and future interfaces. We have categorized the user interfaces into text-based, graphical based and emerging user interfaces.

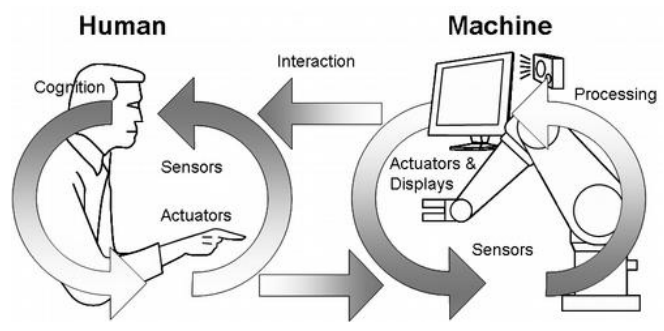

Fig. 1. Example of human machine interaction.

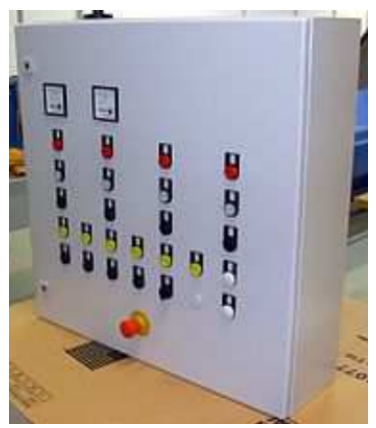

Fig. 2. HMI of sugar industry machine.

The rest of the paper is organized as follows: Sections II, III and IV discuss different categories of user interfaces in detail and finally, the paper is concluded in Section V.

\section{TEXT-BASED INTERfaCES}

\section{A. Batch Interfaces}

It is the non-interactive interface extensively used in the past during the period 1945 to 1968 . In batch interfaces, all jobs or commands are combined in a single batch by the user and executed once instead of executing commands separately. 
All jobs are performed step by step in a given order without any intermediate interaction with the user. The main advantage of this interface is time saving during the interaction period. On the other hand, it is difficult to terminate the execution of running batches until the user completes a job.

\section{B. Command Line Interface (CLI)}

This interface was first time introduced in 1969 and still used by expert programmers. The examples are UNIX, MSDOS application in Windows operating systems, etc. In CLI, the user enters commands on a prompt line through the terminal to interact with the operating system. The response messages from the system are shown on the display screen. It is very tedious to remember different commands as well as command line switches (see Fig. 3).

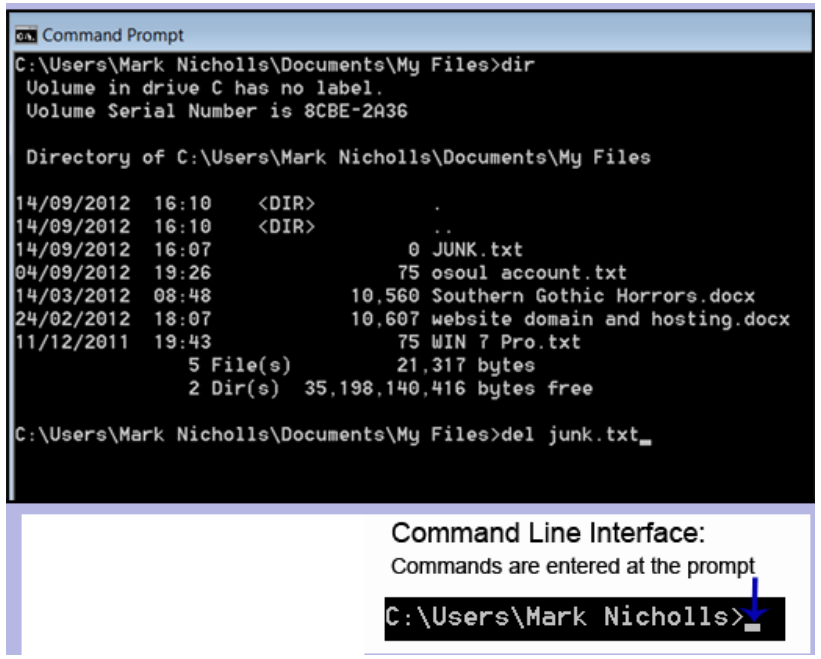

Fig. 3. Example of CLI.

\section{Non-Command User Interface}

In this interface, the computer observes the requirement to understand the user without typing of commands. The interface just shows the alphabet options to type instead of typing complete commands (see Fig. 4).

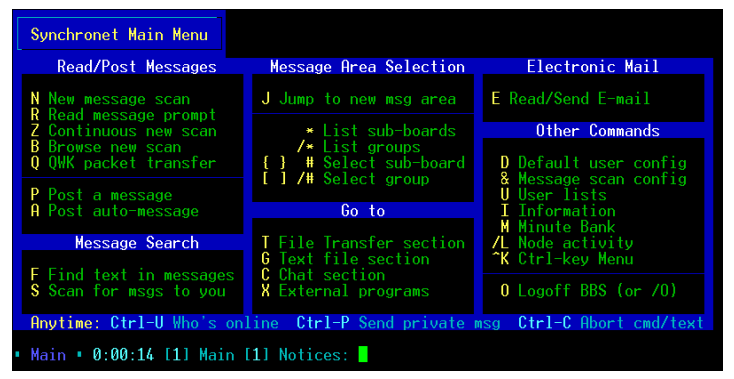

Fig. 4. Example non-command user interface.

\section{Natural Language Interface (NLI)}

This type of interface usually used in the search engines. When the user enters a question using verbs, clauses, and phrases, the response is given to the user after searching the web. In the latest research of relational databases, a query in natural English language is an example of NLI, where the sentence is translated into Structured Query Language (SQL) query using joins, nesting, and aggregation. The SQL query is then entered in relational database management systems and finally, the response is presented to the user. It is convenient for a layman with no knowledge of SQL [2].

\section{GRAPHICAL INTERFACES}

\section{A. Graphical User Interface (GUI)}

The first GUI or WIMP (Windows, icons, mouse pointer) was designed by Xerox Corporations Palo Alto Research Centre in the 1970s, but unfortunately, it did not get popularity till 1980. It was popularized for the first time by the Apple company and then extensively used by the Microsoft Windows (see Fig. 5). Using a well-designed GUI, the user can get benefits in terms of fast learning and ease to use. Examples are Microsoft Windows, Apple Macintosh, and Ubuntu operating systems. For UNIX-based systems, both CLI and GUI interfaces are used.

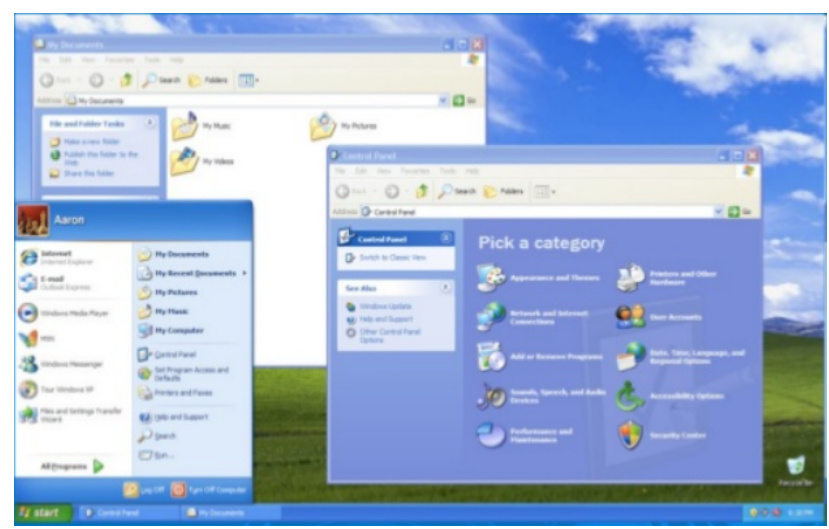

Fig. 5. Example of GUI.

\section{B. Attentive User Interface (AUI)}

In AUI, the notifications or messages are shown to the user and asked to enter the same information to ensure his presence for security purposes before generating related information [3]. The examples are Captcha entry, session expiry, etc. (see Fig. 6).

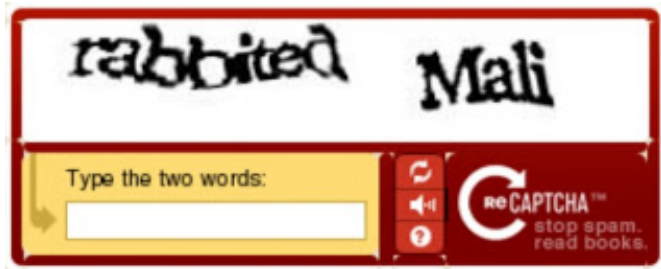

Fig. 6. Example CAPTCHA: The user is asked to type the printed words before proceeding.

\section{Crossing-Based Interface}

In this interface, the cursor or pointer is moved in or out from a graphical object area e.g., hyperlink on mouse hover. 


\section{Intelligent User Interface (IUI)}

This interface deals with artificial intelligence having domain knowledge based on reasoning to assist the users [4], [5]. It has the capability "to adapt the user, communicate with the user, and solve problems for the user", e.g., office assistant or wizard, etc.

\section{E. Reflexive User Interfaces}

In this interface, the user controls the whole system and can redefine its commands or define new commands by performing actual actions using a rich graphical interface.

\section{F. Touch User Interface (TUI)}

The TUI is a special kind of GUI using a touch-pad or a touch-screen display as a combined input and output device. In TUI, the display screen is pressure sensitive where the user interacts with the machine by tapping the screen surface using fingers or stylus. On tapping, the system compares the actions stored in the database and executes appropriate commands. Fig. 7 shows an example of TUI. Currently, the TUI is widely used in mobile devices, point of sales, industrial processes, and self-service machines, etc.

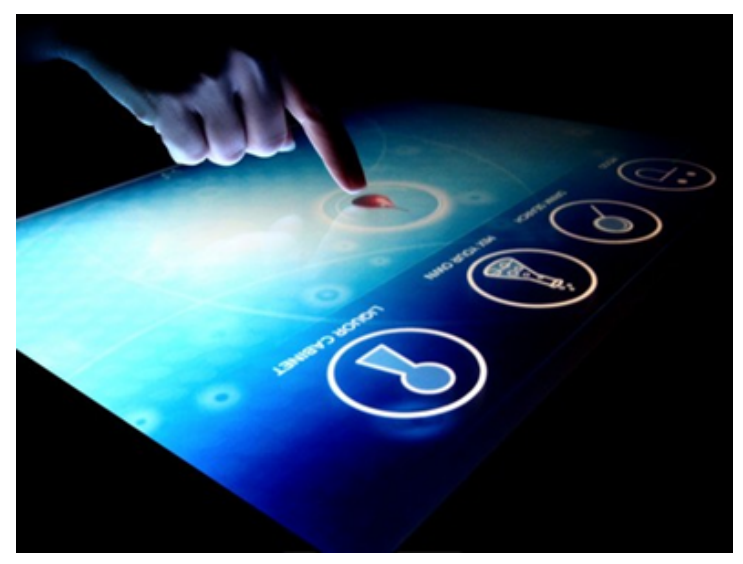

Fig. 7. Example of touch screen user interface.

\section{G. Tangible User Interface}

In this interface, the user interacts with the digital information using the physical environment [6]. In this way, physical movements are translated into digital information. The simplest example is the mouse movement to control arrow pointer on the display screen. Microsoft has also introduced a tangible user interface-based Windows platform, Microsoft Surface (new name is Microsoft PixelSense) in the year 2000.

\section{H. Conversational Interface Agents}

In this interface, messages, voices, chatbots with plain English text are presented to the user with the help of animated character, robot or person just like Microsoft Clippy for emulating human to human conversation. ${ }^{2}$

${ }^{2}$ http://www.cbc.ca/news/canada/toronto/toronto-chatbots-1.3581791

\section{Multi-Screen Interface}

To provide more flexible interaction with the user, multiple screens are used. It is normally used in hand-held markets and computer games to show different statistics at multiple displays of stock markets, etc. (Fig. 8).

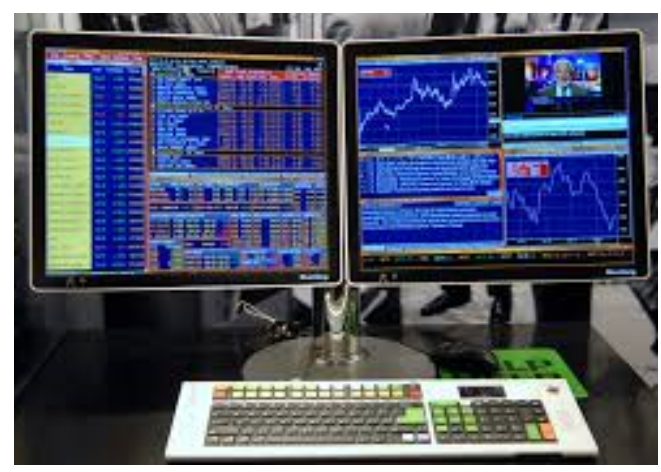

Fig. 8. Examples of multiscreen user interface.

\section{J. Keyboard Driven Modeless User Interface}

In this interface, keyboard shortcuts are used instead of typing command or clicking the options on toolbar or menu bar. Using keyboard shortcuts, the input speed is tremendously increased. $^{3}$

\section{K. Zooming User Interfaces (ZUI)}

The ZUI is pronounced as zoo-ee in a graphical environment, in which user can zoom in or zoom out to view more or fewer details (see Fig. 9).

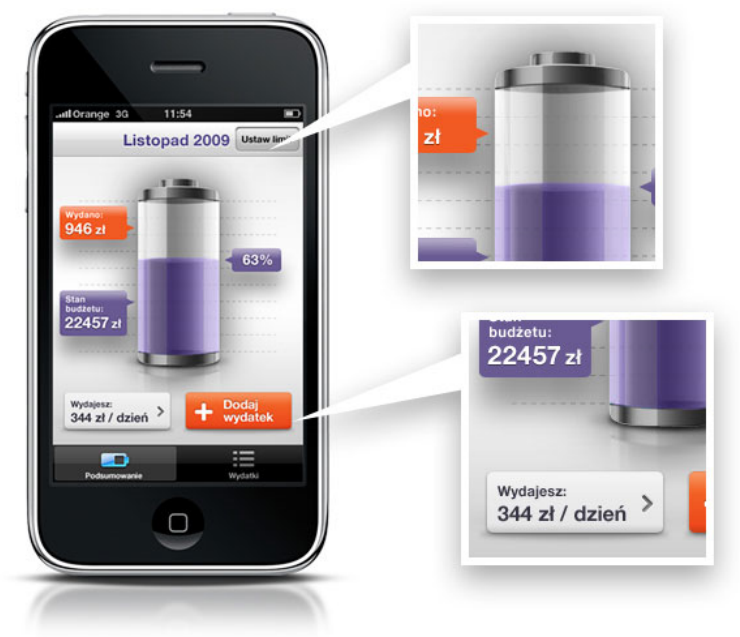

Fig. 9. Examples of Zooming UI.

${ }^{3}$ https://blog.mozilla.org/labs/2007/07/the-graphical-keyboard-userinterface/ 


\section{Menu Driven Interface}

It is a subtype of GUI in which applications have a menu bar with different menu items. By clicking the menu items or by moving the Mouse on these menu items, commands are executed (Fig. 10).

In the next section, we discuss all kinds of latest devices and interfaces where user interactions are not possible using commands or menus, icons, etc.

\begin{tabular}{|c|c|c|c|}
\hline & & Untitled - My App & - $\square x$ \\
\hline \multicolumn{4}{|c|}{ File Edit View Tools Help } \\
\hline & New & $\mathrm{CTRL}+\mathrm{N}$ & \\
\hline & Open & $C T R L+O$ & \\
\hline & Save & $\mathrm{CTRL}+\mathrm{S}$ & \\
\hline & Save As... & & \\
\hline & Page Setup... & & \\
\hline & Print... & $C T R L+P$ & \\
\hline & Exit & & \\
\hline
\end{tabular}

Fig. 10. Example of menu driven interface.

\section{EMERGING USER INTERFACES (EUI)}

There exist situations where the interaction with computers and machines is achieved by observing the user's body relative movements, direction of gaze, or gestures and respond to the specific human action accordingly without executing of commands or mouse clicks. It is achieved with the help of sensors for tracking the positions of body parts. The examples are Sixth Sense device, Google Glass, and gestures of tracking pad, magic mouse.

\section{A. Eye Movement-based Interface}

In this interface, natural eyes are used as input for HCI. It is not using windows icon, menus and is highly interactive. It is natural and needs little conscious efforts. "What You Look At is What You Get". But it needs efficient recognition algorithms for eye-movements.

\section{B. Brain-Computer Interface}

The use of mouse and keyboard for HCI is not suitable for disabled users. It uses brain signals similar to the eyemovements based interaction to communicate with the machines and computers.

\section{Gesture User Interface}

Gestures are already being used in human conversations along with the speech for better understanding the conversation. Similarly, to interact with the computers or machines, gestures are also used. In this type of interaction, the input is accepted through human gestures like hand gestures, or mouse gestures received using a computer mouse/stylus [7] (Fig. 11). Gestures are captured with the help of cameras and stored in the database. When gestures are captured using input devices such as scanners or cameras, the processor inside these devices compares with the database and performs action accordingly [8].

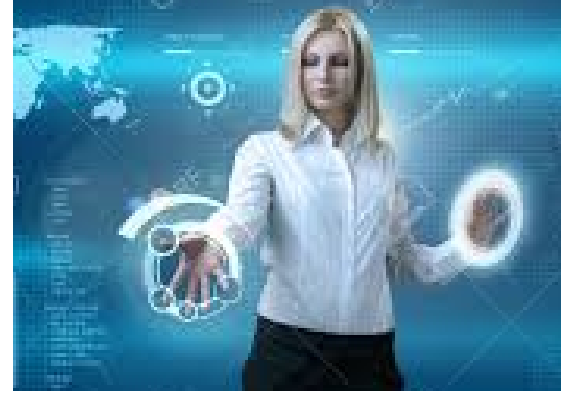

Fig. 11. Examples of gesture-based interaction.

\section{Motion Tracking Interfaces}

In this interface, human body movements are captured and translated into commands. It was first time introduced by Apple and the latest one uses a camera for this purpose [9].

\section{E. Voice User Interface}

In this interface, the audio voice input is given to the system and after voice recognition, appropriate action is performed followed by voice prompts (Fig. 12). The examples are Siri application (APP) in Apple mobile phone, Google Glass, Cortana, Amazon Echo (Alexa), Google Now, Jibo Assistant.ai, and Ubi. Fig. 13 shows examples of interaction with the computer or machine using voice signals coming from the microphone. (a)

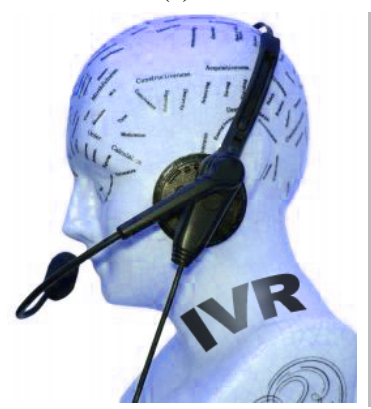

(b)

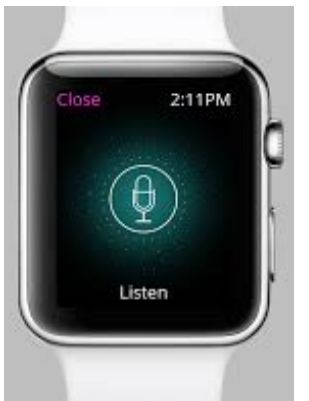

Fig. 12. Examples of voice user interfaces.

\section{F. Zero-Input Interfaces (ZII)}

This interface is used for direct input to electronic circuits from sensors for HCI interaction rather than typing commands by the user.

\section{G. The SixthSense Device}

The SixthSense device is developed by Pranav Mistryis, a PhD student in the Fluid Interfaces Group at MITs Media Lab. The SixthSense device is used to interact with physical objects using human gestures. ${ }^{4}$ If we consider inside the computer as a digital world and outside the computer as a physical world, the interface between the physical and digital worlds provides connections between objects and gestures. The

\footnotetext{
${ }^{4}$ https://tedsummaries.com/2014/03/18/pranav-mistry-the-thrillingpotential-of-sixthsense-technology/
} 


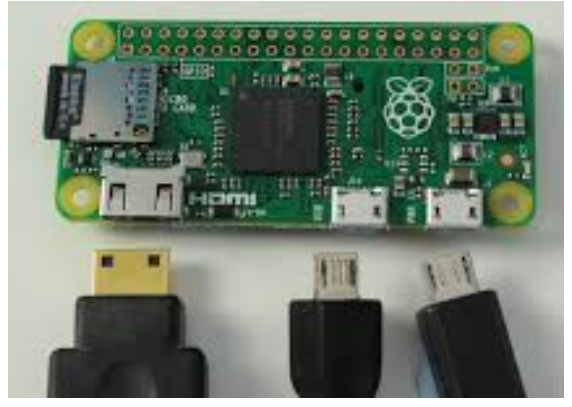

Fig. 13. Examples of interaction with the computer or machine using voice signals.

communication with the computer is achieved in a supernatural manner using gestures. The keyboard amazingly appears on hand and touching the figures on hand, the user can access the computer system (see Fig. 14(a)). Amazingly, a person is recognized by its profession as he appeared before the system (see Fig. 14(b)). Observing the time is amazingly achieved by drawing gesture of watch on the forehand and the watch appears (see Fig. 14(c)).

The idea was to combine both physical and digital worlds together. The device is always connected to the internet. Whenever user holds a book, the book's audio version is searched on the internet and the user can listen the audio of the book. Amazingly, playing the live talk of former American President Mr. Obama, just by reading the online news from the internet with the help of SixthSense device. Another interesting idea is playing games on paper (rotating or moving the paper control the car movements) and displaying some pictures on paper as well as printing the hard copy of pictures by touching the print button on paper (see Fig. 15). (a)
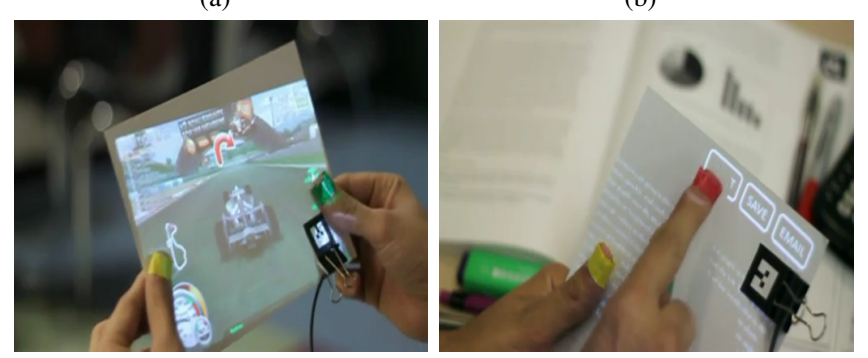

Fig. 15. Example SixthSense device (a) playing games and displaying on paper (b) printing from paper.

\section{H. Google Glass}

The Google Glass is an amazing wearable small gadget connected with the internet and interacts with the computer without commands or graphical objects. It is head mounted and shows information to the user without touching with hands [10], [11]. It contains a built-in camera, touchpad, and voice recognition software. It can take photographs and record videos as well as displaying to the users in front of their eyes. It has built-in Wi-Fi for surfing the internet and sending emails. It has built-in memory to store media files and messages. Fig. 16 shows an example of wearing Google Glass where the output is displayed in front of the eyes.
The Google Glass helps in internet surfing and searching data by sending verbal messages. It also records the daily activities of the user wearing it, and assist in various circumstances such as suggesting alternate routes in case of traffic congestion on a road. The communication with different languages is also possible using Google translate feature supported in Google Glass. (a)

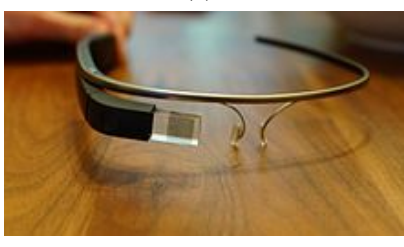

(b)

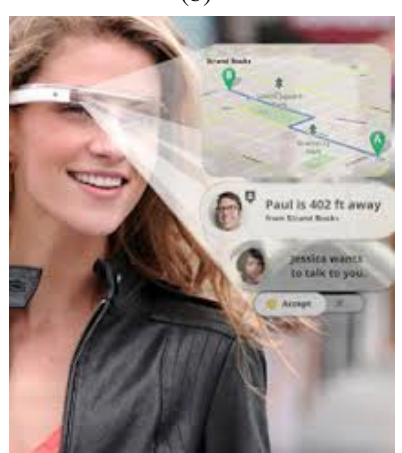

Fig. 16. Example Google Glass User Interface (a) Google Glass Gadget (b) Wearing Google Glass and display the output in front of the eyes.

\section{Muse Headband}

The Muse Headband is a mind reading device containing four sensors to read and extract information from mind waves. ${ }^{5}$ The aim is to apply the human thinking in a computer such as changing the text font, playing games, printing documents on computers without physical interactions [12]. This interface may result in reducing the brain stress to remember or executing different commands. Fig. 17 shows the brain waves captured with the help of headband (right side). The text font is changed automatically, the moment, the idea of changing the font comes to the user's mind (left side). (a)

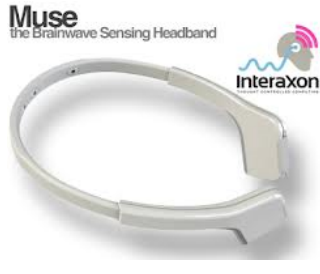

(b)

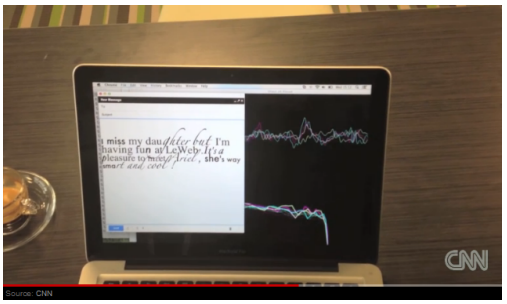

Fig. 17. Example (a) Muse Head Band (b) Muse Head User Interface to change the text font due to thinking only.

\section{CONCLUSiON}

In this paper, an overview of existing user interfaces is provided. The user interfaces are grouped into three broad categories i.e., text-based, graphical, and emerging user interfaces. Among different user interfaces, the most commonly used ones are the CLI and the GUI. The CLI requires user training and it is tedious and laborious. Whereas, the GUI is simple and it requires minimal training. Recently, some new devices are invented which do not use the conventional user interfaces

\footnotetext{
${ }^{5}$ http://edition.cnn.com/2012/12/06/tech/ariel-garten-muse-interaxon/index.
} $\mathrm{html}$ ?iref=allsearch 
(a)

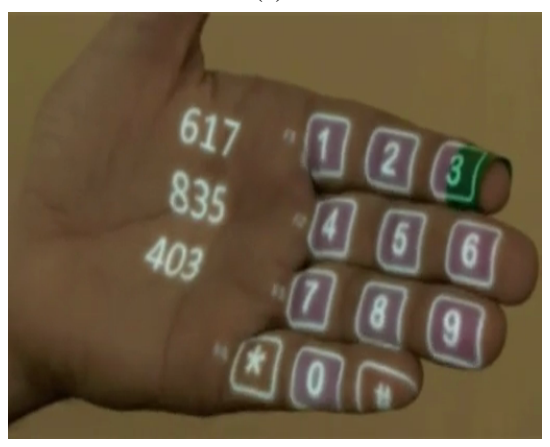

(b)

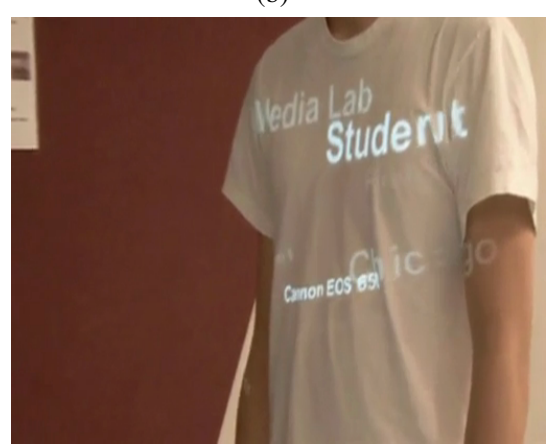

(c)

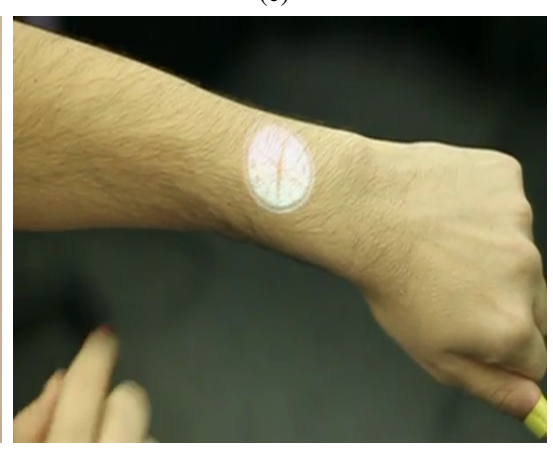

Fig. 14. Example SixthSense device (a) displaying keypad on palm (b) A person coming before this system recognized as student (c) watch on hand.

to interact with the machines and computers. Text based interfaces still being enhanced like Natural language interfaces. Touch screens have played an important role in mobile phone market. Gesture based interfaces have also important impact in the gaming industry. The Emerging user interfaces have a big share in the market of tablets, smartphones, television, gaming consoles, Google Glass, sixth sense devices, etc. In future, it is most likely the EUI may become famous just like the GUI. Using EUI, the integration of information with various devices will help human to be more connected to the physical world. It will eventually help in staying for a long time in front of machines. The survey will be helpful in designing new interfaces for interaction between the machines and computers.

\section{REFERENCES}

[1] A. Razzaq, "A new emerging interface: Sorcerous user interface sui," Researcher, vol. 7, no. 5, 2015.

[2] F. Li and H. V. Jagadish, "NaLIR: an interactive natural language interface for querying relational databases," in Proceedings of the 2014 ACM SIGMOD international conference on Management of data. ACM, 2014, pp. 709-712.

[3] B. A. Huberman and F. Wu, "The economics of attention: maximizing user value in information-rich environments," Advances in Complex Systems, vol. 11, no. 04, pp. 487-496, 2008.
[4] M. Maybury, "Intelligent user interfaces: an introduction," in Proceedings of the 4th international conference on Intelligent user interfaces. ACM, 1998, pp. 3-4.

[5] M. T. Maybury and W. Wahlster, Readings in intelligent user interfaces. Morgan Kaufmann, 1998.

[6] H. Ishii, "The tangible user interface and its evolution," Communications of the ACM, vol. 51, no. 6, pp. 32-36, 2008.

[7] M. A. Qureshi, A. Aziz, A. Saeed, M. Hayat, and S. Rasool, "Implementation of an efficient algorithm for human hand gesture identification," in Electronics, Communications and Photonics Conference (SIECPC), 2011 Saudi International. IEEE, 2011, pp. 1-5.

[8] J. Rico, A. Crossan, and S. Brewster, "Gesture-based interfaces: practical applications of gestures in real world mobile settings," in Whole Body Interaction. Springer, 2011, pp. 173-186.

[9] S. Fateh, "Motion detection and tracking system to control navigation and display of portable displays including on-chip gesture detection," Sep. 12 2005, uS Patent App. 11/225,877.

[10] T. Starner, "Project glass: An extension of the self," IEEE Pervasive Computing, vol. 12, no. 2, pp. 14-16, 2013.

[11] D. Pogue, "Google glass and the future of technology," The New York Times, 2012.

[12] O. E. Krigolson, C. C. Williams, and F. L. Colino, "Using portable eeg to assess human visual attention," in International Conference on Augmented Cognition. Springer, 2017, pp. 56-65. 